\title{
Magnetization Reversal of Modified Circular Nanoscale Ferromagnetic Elements
}

\author{
H. Hu*, H. Wang**, M. R. McCartney*, David J. Smith* \\ *Department of Physics and Astronomy, Arizona State University, Tempe, AZ 85287-1504 \\ ${ }^{* *}$ Dept. of Chemical and Materials Engineering, Arizona State University, Tempe, AZ 85287-6006
}

In recent years, submicron magnetic rings have attracted much attention because of their potential applications for magnetic random access memory (MRAM) and magnetosensors [1]. One of the requirements for such a memory application is that the remanent magnetic state be bi-modal. Circular elements have potential for use in memory or sensor devices given their natural tendency to form vortex-like magnetic configurations, in either clockwise or counter-clockwise directions. Additional considerations involve the amount of fringing fields, which impacts the packing density of the array and the ease with which the element can be reversed.

In this work we present experimental studies and theoretical magnetic simulations investigating the switching behavior of patterned ferromagnetic elements using off-axis electron holography [2]. This technique enables quantitative information about magnetic fields to be obtained with nanometerscale resolution, In addition, we are able to do in situ observation of the magnetization reversal process within the electron microscope. $10 \mathrm{~nm}$-thick polycrystalline Co elements were fabricated by electron beam lithography and lift-off process. The outer diameters (OD) of these circular elements were $400 \mathrm{~nm}$ and $300 \mathrm{~nm}$.

The magnetization reversal behavior of cut-off rings (COR) with different inner diameters (ID) were compared with those of complete rings with similar sizes. Complete rings showed very different switching behavior depending on their OD/ID ratios. In one case, the reversal started from the socalled onion state, proceeded to a stable vortex state, and finally to a reversed onion state (Fig. 1). Alternatively, the reversal was accomplished via a non-vortex switching process: two domain walls of the onion state moved around the rings coherently without the formation of a vortex state (Fig. 2). The in-plane magnetization direction could be identified by measuring the phase gradient from the reconstructed phase images (not shown here). The switching field range, which was the difference between the feld value of the original and reversed onion state, was very large, typically several hundred Oe. When the applied field direction was perpendicular to the slots, well-defined single domain states were identified at remanence in the cut-off disks and rings (Fig. 3). Cut-off disks showed a vortex state when switched between two single domain states. Cut-off rings, on the other hand, showed a strong likelihood of non-vortex switching. The switching field range of a single element (less than 40Oe) was much smaller than a complete ring. Moreover, the switching field range was not dependent on size or slot angle. The element-to-element interactions were very small for cut-off rings with small slot angles $\left(30-60^{\circ}\right)$ because of the small stray field around each element. This shape could be advantageous for highly-packed information storage applications and MRAM design.

\section{References}

[1] J.G.Zhu, Y.Zheng, and G.A.Prinz, J. Appl. Phys. 87, 6668 (2000).

[2] H. Hu, H. Wang, M. R. McCartney and D. J. Smith, J. Appl Phys, in press.

[3] Support for this research was provided by the ONR \#.N00014-02-1-0922. 


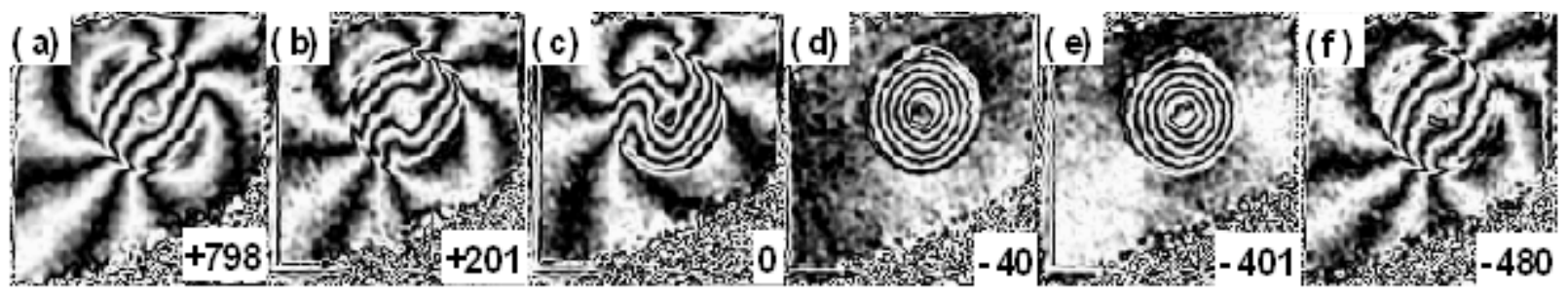

FIG. 1. Amplified (8x) phase images of $400 \mathrm{~nm} / 50 \mathrm{~nm}$ rings during half of a hysteresis cycle showing a vortex-switching process. The applied field was along the diagonal from top right to bottom left. In-plane field magnitudes are indicated below each image (units: Oe). The black and white lines are parallel to the in-plane magnetic induction. Vortex states are visible in (d) and (e). A "U"-shaped state is visible in (c).

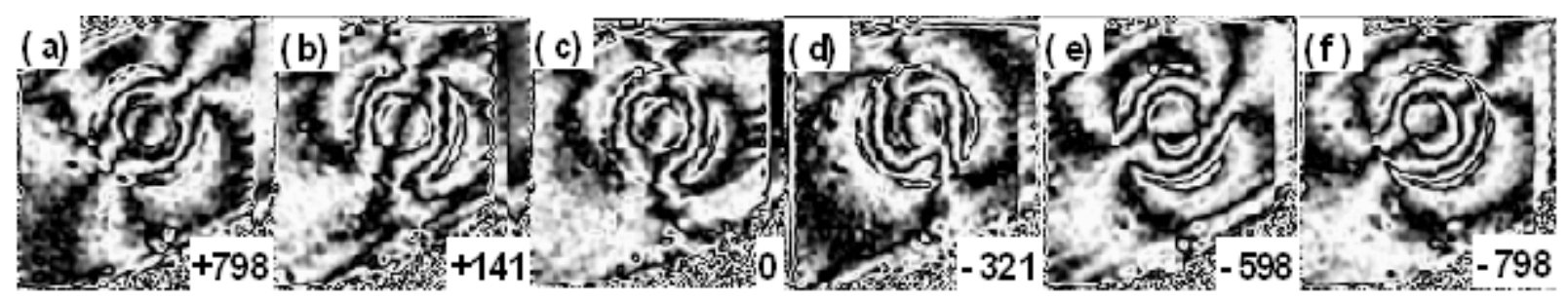

FIG. 2. Amplified (8x) phase images of $400 \mathrm{~nm} / 150 \mathrm{~nm}$ rings during half of a hysteresis cycle showing a coherent domain wall rotation process. The applied field was along the diagonal from top right to bottom left. In-plane field magnitudes are indicated below each image (Units: Oe). The black and white lines are parallel to the in-plane magnetic induction. Two domain walls move coherently around the rings. No vortex is formed.

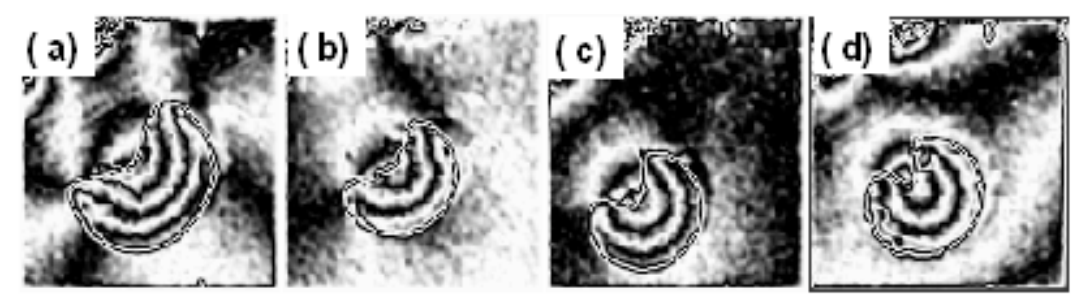

FIG. 3. Amplified (4x) phase images of remanent states of (a) 400nm, $120^{\circ}$ cut-off disk (COD); (b) a $300 \mathrm{~nm}, 120^{\circ} \mathrm{COD}$; (c) a $300 \mathrm{~nm}, 90^{\circ} \mathrm{COD}$ and (d) a $300 \mathrm{~nm}, 60^{\circ} \mathrm{COD}$. The applied field was along the diagonal from top right to bottom left. The black and white lines are parallel to the inplane magnetic induction. All shapes show stable "single domain" states. 